\title{
Simulations of Cosmic Chemical Enrichment
}

\section{Chiaki Kobayashi}

National Astronomical Observatory of Japan; chiaki@th.nao.ac.jp

\begin{abstract}
We simulate cosmic chemical enrichment with a hydrodynamical model including supernova and hypernova feedback. We find that the majority of stars in present-day massive galaxies formed in much smaller galaxies at high redshifts, despite their late assembly times. The hypernova feedback drives galactic outflows efficiently in low mass galaxies, and these winds eject heavy elements into the intergalactic medium. The ejected baryon fraction is larger for less massive galaxies, correlates well with stellar metallicity. The observed mass-metallicity relation is well reproduced as a result of the mass-dependent galactic winds.
\end{abstract}

\section{Hypernovae}

Supernovae eject not only thermal energy but also heavy elements into interstellar medium. Thus the star formation history is imprinted in the chemical abundances of stars and galaxies. Different types of supernovae, i.e., Type II and Ia supernovae (SNe II and Ia), produce different heavy elements with different timescales. Recently, it is found that hypernovae $(\mathrm{HNe})$, which have more than ten times larger explosion energy, produce a certain amount of iron. Kobayashi et al. (2006a) calculated the nucleosynthesis yields for wide ranges of metallicity and energy, based on the light curve and spectra fitting of individual supernovae. With these yields, the elemental abundance ratios are in good agreement with observations from oxygen to zinc. Especially, the observed abundance of $\mathrm{Zn}([\mathrm{Zn} / \mathrm{Fe}] \sim 0)$ can be explained only by a large contribution of HNe.

\section{Cosmological Simulations}

We simulate the evolution of gas and stellar systems and the chemical enrichment from SNe II, SNe Ia, and HNe, from the cosmological initial condition with $H_{0}=70 \mathrm{~km} \mathrm{~s}^{-1}$ $\mathrm{Mpc}^{-1}, \Omega_{m}=0.3, \Omega_{\Lambda}=0.7, \Omega_{\mathrm{b}}=0.04, n=1$, and $\sigma_{8}=0.9$ (see Kobayashi, Springel \& White 2006b for the details). We use an SPH code GADGET-2 by Springel (2005), and introduce the metal-dependent cooling rates (Sutherland \& Dopita 1993) and chemical enrichment scheme by Kobayashi (2004). The initial condition is calculated in a $10 h^{-1}$ Mpc cubic box with periodic boundary conditions with $N_{\mathrm{DM}}=N_{\text {gas }}=96^{3}$. With the HN feedback, the cosmic SFRs shows a peak at $z \sim 4$, with $\sim 10 \%$ of baryons turning into stars, roughly consistent with observational estimates (Fukugita \& Peebles 2004).
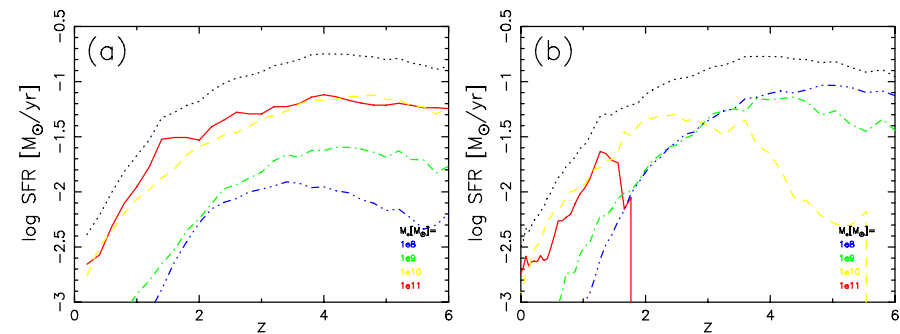

Figure 1. The cosmic SFR for the different galaxy mass with the stellar mass of $10^{11}$ (solid line), $10^{10}$ (dashed line), $10^{9}$ (dot-dashed line), and $10^{8} M_{\odot}$ (dot-dot-dot-dashed line). The dotted line shows the total. Galaxies are identified by FOF at $z=0$ (a) and at each redshift (b), respectively. 

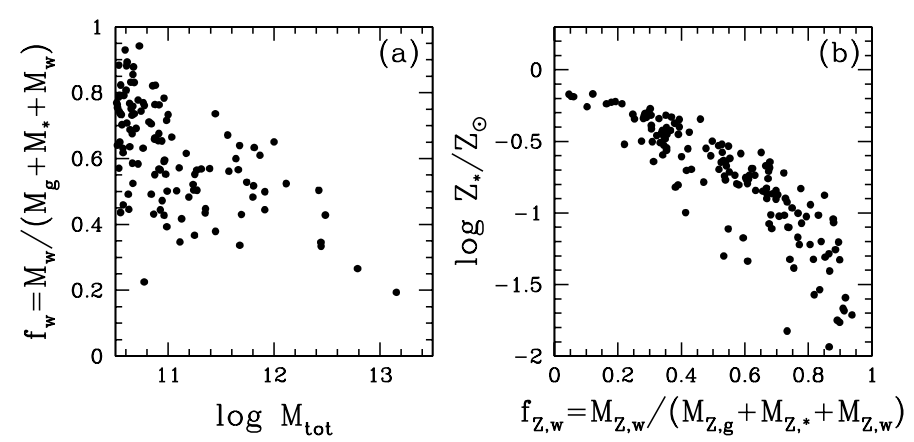

Figure 2. The wind fraction against the total mass (a), and the stellar metallicity against the ejected metal fraction (b).

When and where do stars form? To answer this question, we break up the cosmic SFR history according to galaxy mass. In Fig. 1a, the galaxies have been identified by FOF at $z=0$ for this plot, and thus these SFRs correspond to the age distribution of stars in the galaxies. For all galaxy masses, the SFRs show a peak around $z \sim 3-4$, and the majority of stars are as old as $\sim 10$ Gyr. On the other hand, in Fig. 1b, we identify galaxies at each redshift, which are comparable to the observations of high redshift galaxies. This shows that most stars have formed in low-mass galaxies with $10^{8-9} \mathrm{M}_{\odot}$ at high redshift $z \gtrsim 3$. $10^{10} M_{\odot}$ galaxies exist at high redshift $z \lesssim 5$, but $10^{11} M_{\odot}$ galaxies appear only after $z \sim 2$. From these two figures we conclude that most stars have formed in dwarf galaxies before they merge to massive galaxies in our simulation. As a result of the hierarchical clustering of dark matter halos, such old stars belong to massive galaxies at low redshifts.

How are heavy elements ejected from galaxies to the IGM? In the simulation, we can trace the orbit of gas particles over time. Exploiting this, we define as wind particles those that are not in galaxies now, but have been in galaxies before. In this simulation, $\sim 10 \%$ of baryons turn into stars, $\sim 10 \%$ of the gas stays in galaxies ( $\sim 8 \%$ is hot), and $\sim 20 \%$ is ejected as galactic winds. The rest, half of the baryons, never accretes onto galaxies. We can also examine from which galaxies the wind gas particles are ejected. This allows a measurement of the ejected wind mass from each galaxy. In Fig. 2a, a clear relation is found between the wind fraction and the total mass. Winds are efficiently ejected from small galaxies. A similar relation is also found for the ejected metal fraction. It is interesting that the wind fraction and the ejected metal fraction correlate well with the stellar metallicity (Fig. 2b). Based on this finding, we conclude that the origin of the mass-metallicity relation can be explained with galactic winds.

The metal enrichment timescale depends on the environment. In large galaxies, enrichment takes place so quickly that $[\mathrm{O} / \mathrm{H}]$ reaches $\sim-1$ at $z \sim 7$, which is consistent with the sub-solar metallicities of the Lyman break galaxies (Pettini et al. 2001). The low metallicities of DLA systems (Prochaska et al. 2003) are also consistent with our galaxies, provided these systems are dwarf galaxies or the outskirts of massive galaxies. The low $[\mathrm{C} / \mathrm{H}]$ of the IGM (Schaye et al. 2003) can be explained if the IGM is enriched only by SNe II and HNe. The average metallicity of the universe reaches $[\mathrm{O} / \mathrm{H}] \sim-2$ and $[\mathrm{Fe} / \mathrm{H}] \sim-2.5$ at $z \sim 4$, but reaches the same values at $z \sim 3$ in the IGM.

In galaxies, metallicity of the cold gas increases with galaxy mass, which is comparable to observations with a large scatter (Tremonti et al. 2004). The central cold gas shows a relation between galaxy mass and metallicity with shallower slope than observed in emission-line galaxies. For the stellar population, the observed mass-metallicity relation is well reproduced (Kobayashi \& Arimoto 1999; Pahre et al. 1998; Gallazzi et al. 2005), and originates in mass-dependent galactic winds. These relations are present since $z \sim 5$. 